\title{
Dyke Davidoff Mason Syndrome Presenting as Hemiparesis and Refractory Status Epilepticus: A Case Report
}

\section{Annapurna Rai, Utpal Kant Singh and Rajniti Prasad*}

Department of Pediatrics, Institute of Medical Sciences, B.H.U, Varanasi, India

*Corresponding Author: Rajniti Prasad, Department of Pediatrics, Institute of Medical Sciences, B.H.U, Varanasi, India.

Received: September 30, 2019; Published: October 15, 2019

DOI: 10.31080/ASNE.2019.02.0117

\begin{abstract}
Dyke Davidoff Mason syndrome is characterized by facial asymmetry, contralateral hemiplegia, mental retardation and seizures due to atrophy or hypoplasia of cerebral hemispheres resulting from insult to developing brain. Here we report a-2yrs-male presented with hemiparesis, global delay and refractory status epilepticus and later diagnosed as Dyke Davidoff Mason syndrome.
\end{abstract}

Keywords: Dyke Davidoff Mason Syndrome (DDMS); Hemiparesis; Seizure; Global Delay

\section{Introduction}

In Dyke Davidoff Mason Syndrome (DDMS) there is atrophy or hypoplasia of one cerebral hemisphere, which occurs due to brain insult in fetal or early childhood period [1]. The various causes are congenital abnormalities, cerebral infarction, vascular malformations, infections, gestational vascular occlusion, primarily involving MCA, birth trauma, hypoxia, intracranial hemorrhage, tumors, prolonged febrile seizures, and coarctation of aorta [1]. A vascular anomaly occurring in early gestation results in major brain development defects compared to those occurring later. The two types of DDMS are congenital (infantile) and acquired [2]. Infantile type is characterized by contralateral hemiplegia, variable degree of facial asymmetry with characteristic skull changes, mental retardation, and seizures $[3,4]$. In this condition, there is shift of midline structures towards the side of disease with absent sulcal prominence in congenital type, whereas in acquired type sulcal spaces are prominent [5]. We are presenting here a case of a 2 years male child of DDMS with refractory seizure, hemiparesis and global delay.

\section{Case Report}

A-2-years male had presented in pediatric outpatient department with complaints of weakness in left side of body observed by parent since 9 months of age, inability to stand and speak and abnormal movement of all four limbs for more than 30 minutes. In emergency, after securing airways, intravenous lorazepam $(0.1 \mathrm{mg} /$ $\mathrm{kg}$ ) was given followed by intravenous phenytoin (loading $20 \mathrm{mg} /$ $\mathrm{kg})$, levetiracetam $(20 \mathrm{mg} / \mathrm{kg}$ as bolus and repeated after $20 \mathrm{minu}-$ tes) and midazolam infusion $(6 \mu \mathrm{g} / \mathrm{kg} /$ minute to control seizure) followed by maintenance dose of phenytoin and levetiracetam.

There was no history of head trauma, any consanguinity and psychiatric manifestation. The history of birth asphyxia was present. After cessation of seizure, vitals were stable and there was weakness of left side, tone and deep tendon reflexes were exaggerated and positive Babniski's sign. The power in left side, power was $3 / 5$ in left upper and lower limb and is 5/5 in right side on day 4 of admission. Patient had facial asymmetry. Child had global developmental delay (neurodevelopmental score: motor and adaptive $<20$, language: 60, personal social: 40). Examination of cardiovascular, respiratory and abdomen were unremarkable.

Complete blood counts were normal, renal and liver function tests were normal. Examination of cerebrospinal fluid was normal [cells:5/mm3 (lymphocyte only), glucose:86 mg/dL (blood glucose:108 mg/dL) and protein: $36 \mathrm{mg} / \mathrm{dL}$. Blood and Cerebrospinal fluid culture were sterile. MRI of brain reveals right sided atrophic cerebral hemispheres, basal ganglia, thalamus, cerebellum and brainstem with prominent sulci and associated changes secondary to previous ischemic insult, feature was suggestive of Dyke-Davidoff-Mason syndrome

(Figure a-c). Radiograph of sinuses was not done. 

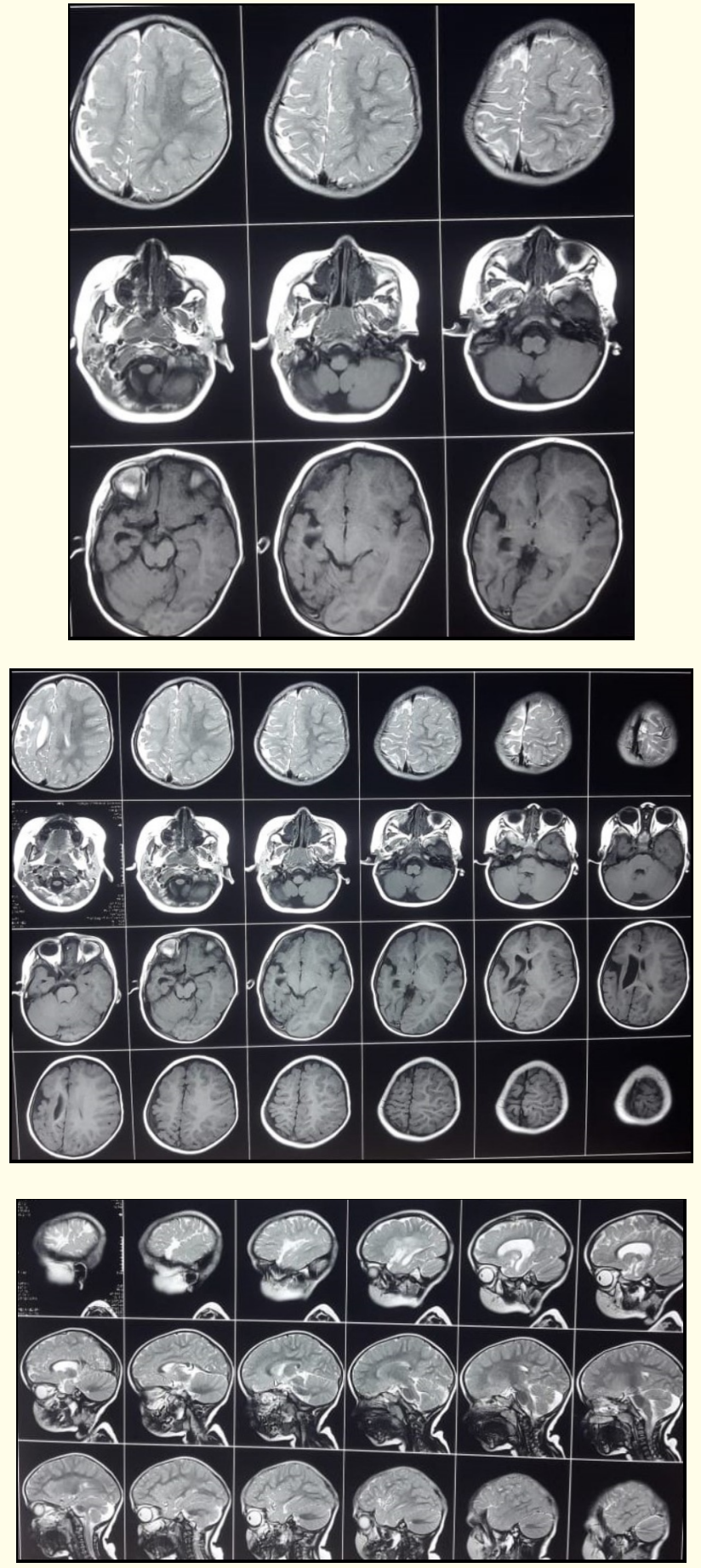

Figure 1 a, b \& c: MRI of brain showing moderate atrophy of right cerebral hemisphere with prominent right ventricle with atrophied right thalamus, brainstem and cerebellum with paucity of white matter.

\section{Discussion}

In 1993,Dyke, Davidoff and Mason illustrated radiographic and pneumatoencephalographic changes such as thickened calvarium and dilatation of ipsilateral frontal and ethmoid sinuses with elevation of greater wing of sphenoid and petrous ridge in nine patients, who presented with hemiparesis, seizures, facial asymmetry and mental retardation [6]. They hypothesized that these changes were secondary to congenital/acquired brain insults.

Three MRI patterns are described by Shen., et al. Pattern I showing diffuse cortical and subcortical atrophy, Pattern II showing diffuse cortical atrophy with porencephalic cysts and pattern III showing previous infarction with gliosis in middle cerebral artery territory $[7,8]$. The vascular occlusion or malformation involving middle cerebral artery (MCA) is the most common cause which manifests as hemiplegia at birth or early infancy. There are other causes including infections, trauma, prolonged febrile seizures, or ischemic/hemorrhagic insults early in life.

The differential diagnosis include Rasmussen encephalitis, Sturge Weber Syndrome, Fishman syndrome, hemimagalencephaly, Miller Fisher Syndrome, basal cell germinoma, Silver Russel syndrome, linear nevus syndrome $[9,10]$. An appropriate clinical history, and CT or MRI brain provides clinical history are useful in differentiating congenital and acquired DDMS.

The treatment is symptomatic, and includes control of seizure, physiotherapy for hemiplegia, and speech therapy for learning difficulties $[11,12]$. The prognosis depends upon the age and frequency of seizure episodes. The child needs hemispherectomy if intractable seizures and disabling hemiplegia [11,12]. Later child may develop sensory or psychiatric manifestations like Schizophrenia.

\section{Bibliography}

1. Sharma S., et al. "Dyke-Davidoff-Masson Syndrome". Indian Journal of Radiology and Imaging 16 (2006): 165-166.

2. Pendse NA., et al. "Dyke-Davidoff-Masson syndrome (DDMS)". Indian Journal of Pediatrics 71 (2004): 943.

3. Ono K., et al. "Dyke-Davidoff-Masson Syndrome manifested by seizure in late childhood: A case report". Journal of Clinical Neuroscience 10 (2003): 367-71.

4. Amann B., et al. "Treatment- refractory Schizoaffective disorder in a patient with Dyke-Davidoff Masson Syndrome". CNS Spectrums 14 (2009): 36-39.

5. Shetty DS., et al. "Dyke-Davidoff-Masson syndrome". Neurology India 51 (2003): 136.

6. Dyke CG., et al. "Cerebral Hemiatrophy and homolateral hypertropkhy of skull and sinuses". Surgery, Gynecology and Obstetrics 57 (1933): 588-600. 
7. Unal 0., et al. "Left hemisphere and male sex dominance of cerebral hemiatrophy (DDMS)". Clinical Imaging 28 (2004): 163-165.

8. Shen WC., et al. "Magnetic resonance imaging of cerebral hemiatrophy". Journal of the Formosan Medical Association 92 (1993): 995-1000.

9. Bien CG., et al. "Pathogenesis, diagnosis and treatment of Rasmussen encephalitis: A European consensus statement". Brain 128 (2005): 454-471.

10. Lo W., et al. "Updates and future horizons on the understanding, diagnosis, and treatment of Sturge-Weber syndrome brain involvement". Developmental Medicine and Child Neurology 54 (2012): 214-23.

11. Abdel Razek AA., et al. "Disorders of cortical formation: MR imaging features". American Journal of Neuroradiology 30 (2009): 4-11.

12. Narain NP., et al. "Dyke-Davidoff-Masson syndrome". Indian Pediatrics 45 (2008): 927-928.

Volume 2 Issue 11 November 2019

(c) All rights are reserved by Rajniti Prasad., et al. 